\title{
Specific heat of magnetic and semiconductor quasiperiodic structures
}

\author{
S.B. Jácome ${ }^{a}$, I.P. Coelho ${ }^{a, b}$, D.H.A.L. Anselmo ${ }^{a}$, C.G. Bezerra ${ }^{a, *}$ \\ ${ }^{a}$ Departamento de Física, Universidade Federal do Rio Grande do Norte, 59072-970 Natal-RN, Brazil \\ ${ }^{\mathrm{b}}$ Departamento de Ciências Exatas, Centro Federal de Educação Tecnológica do Maranhão, 65025-001 SãoLuís-MA, Brazil
}

Available online 3 March 2007

\begin{abstract}
We have performed a theoretical study of the specific heat $C(T)$, as a function of temperature, of magnetic and semiconductor quasiperiodic structures. The quasiperiodic structures considered here are constructed according to the Fibonacci, double-period and Thue-Morse quasiperiodic sequences. On one hand, we assume the magnetic structures composed of ferromagnetic films, each one described by the Heisenberg model. On the other hand, we consider semiconductor structures composed of slabs of $\mathrm{AlN}$ and $\mathrm{GaN}$, which are characterized by the dielectric functions $\varepsilon_{A}(\omega)$ and $\varepsilon_{B}(\omega)$, and have thicknesses $d_{a}$ and $d_{b}$, respectively. Our results illustrate the effects of disorder on the oscillatory behavior of the specific heat in the low temperature regime.
\end{abstract}

(C) 2007 Elsevier B.V. All rights reserved.

Keywords: Quasicrystals; Spin waves; Phonons; Thermodynamical properties

\section{Introduction}

In the past decades extensive theoretical and experimental investigations have been carried out for collective excitations of artificially layered systems. These specimens, composed by a wide variety of constituent materials, form an intriguing class of physical structures whose macroscopic properties are subject to design and control by varying the thickness and composition of the layers. Most of the initial studies have been concentrated on the properties of perfectly periodic structures. However, the discovery of quasicrystals, by Shechtman and co-workers [1] in 1984, aroused a great interest in quasiperiodic systems. In a general way, quasiperiodic structures, which can be idealized as the experimental realization of a one-dimensional quasicrystal, are built from the superposition of two (or more) building blocks that are arranged in a desired manner. These systems present a degree of disorder that can be defined as an intermediate state between an ordered crystal (their definition and construction follow purely deterministic rules) and a disordered solid (many of their physical properties exhibit an erratical appearance) [2-4]. Many research groups have studied in detail, from a theoretical perspective, the spectra of various elementary excitations in quasiperiodic structures such as light propagation [5], phonons [6], electronic transmission [7], polaritons [8], etc. All of these systems

*Corresponding author. Fax: +55842153791.

E-mail address: cbezerra@dfte.ufrn.br (C.G. Bezerra). 
present a general property, which is perhaps their most characteristic one, namely a complex fractal spectra of energy, which can be considered as their basic signature [8-12]. From an experimental perspective, the first experimental realization (in quasiperiodic GaAs-AlAs heterostructures) was carried out by Merlin and collaborators [12], and since then they have become a rapidly expanding object of theoretical and experimental research.

From a thermodynamical point of view, Tsallis and collaborators [13], in a seminal work, studied the consequences of a fractal spectrum of energy, by considering the most well-known and simple deterministic fractal geometry (the triadic Cantor set). This set is obtained through the repetition of a simple rule: divide a given segment into three equal parts, and then eliminate the central one. They showed that the specific heat of such a system exhibits a very particular behavior: it oscillates log-periodically around a mean value which is equal to the fractal dimension of the spectrum. Many other articles about the thermodynamical properties of fractal spectra of energy, including connections with natural spectra, were produced afterwards (see Ref. [14] and references therein). Furthermore, we extended the model described by Tsallis et al. in a couple of papers $[15,16]$, in order to study the specific heat of spin-wave modes that propagate in quasiperiodic structures, with new features of the specific heat behavior.

In a very recent work Coronado and Carpena [14] have investigated the effects produced by the presence of disorder in a fractal spectra of energy on the corresponding specific heat properties. They found that there is a competition between fractal properties and disorder, and that this competition affects the specific heat mean value, and the periods and amplitudes of the oscillations of the specific heat around its mean value. On the other hand, it is known from the literature that the most important quasiperiodic sequences, usually applied to construct quasiperiodic superlattices, present different levels of disorder. As a matter of fact, the level of disorder of these sequences can be characterized by the nature of their Fourier spectrum, which may be dense pure point (Fibonacci) and singular continuous (double period and Thue-Morse).

We should remark that specific heat is one of the most important response functions in condensed matter physics. Fortunately, specific heat is a physical observable whose experimental measurements are relatively easy to be performed. Therefore, it is possible to compare experimental results with the theoretical predictions so that we can test the reliability of the physical models. The aim of the present article is a contribution to the understanding of the effects of disorder on the properties of the specific heat even further, considering magnetic and semiconductor superlattices build up according to different quasiperiodic sequences (Fibonacci, double period and Thue-Morse), each one presenting a different degree of disorder.

The plan of this work is as follows. In Section 2 we present our theoretical models which describe the magnetic and semiconductor quasiperiodic superlattices, as well as, the quasiperiodic sequences applied for building up these structures (Fibonacci, double period and Thue-Morse). Later, in Section 3, we calculate the fractal spectra of energy, through the transfer matrix technique, and the corresponding specific heat. We should remark that in our calculations we consider Maxwell-Boltzmann's statistics. Finally, the numerical results and the discussion of their main features are presented in Section 4.

\section{Physical models}

\subsection{Magnetic superlattice}

In this section we describe the first physical system considered in this work: quasiperiodic magnetic superlattices with biquadratic exchange coupling. Here we follow the lines of Ref. [17]. We consider magnetic superlattices composed of $n_{A}$ layers of material $A$ and $n_{B}$ layers of material $B$. Materials $A$ and $B$ are simple cubic Heisenberg ferromagnets with bulk exchange constants $J_{A}$ and $J_{B}$, and lattice constant $a$. The layers of each material are arranged according to a quasiperiodic sequence. The spin quantum numbers of the magnetic moments in each material are $S_{A}$ and $S_{B}$, respectively. In our model we consider that within the bulk of materials $A$ and $B$ the magnetic moments interact by the exchange couplings $J_{A}$ and $J_{B}$, respectively. However, across the interfaces, the magnetic moments interact by the interfacial coupling $\left(J_{I}\right)$. 
The Hamiltonian for the bulk of each component is

$$
\mathscr{H}_{\alpha}=(-1 / 2) \sum_{i, j} J_{\alpha} \vec{S}_{i} \cdot \vec{S}_{j}-g \mu_{B} H_{0} \sum_{i} S_{i}^{z},
$$

where the sum in the first term is over nearest neighbors $j, H_{0}$ is the applied magnetic field in $z$-direction, and $\alpha$ is equal to $A$ or $B$. Also, $g$ is the usual Landé factor and $\mu_{B}$ is the Bohr magneton.

The spin-wave dispersion relation in a magnetic superlattice is found by considering the wave solution inside each material and applying appropriate boundary conditions at the interfaces. The solutions for each material can be written as a linear combination of the positive- and negative-going solutions of the bulk case, i.e.,

$$
S_{i}^{+}=\left\{A_{l} \exp \left[i \vec{k}_{A} \cdot\left(\vec{r}-\vec{r}_{l A}\right)\right]+A_{l}^{\prime} \exp \left[-i \vec{k}_{A} \cdot\left(\vec{r}-\vec{r}_{l A}\right)\right]\right\} \exp (-i \omega t)
$$

in component $\mathrm{A}$, cell $l$, and

$$
S_{i}^{+}=\left\{B_{l} \exp \left[i \vec{k}_{B} \cdot\left(\vec{r}-\vec{r}_{l B}\right)\right]+B_{l}^{\prime} \exp \left[-i \vec{k}_{B} \cdot\left(\vec{r}-\vec{r}_{l B}\right)\right]\right\} \exp (-i \omega t)
$$

in component B, cell $l$. These solutions are linked together using the equation of motion for the operator $S_{i}^{+}=S_{i}^{x}+i S_{i}^{y}$, i.e.,

$$
i \hbar \frac{\mathrm{d} S_{i}^{+}}{\mathrm{d} t}=\left[S_{i}^{+}, \mathscr{H}\right]
$$

The boundary conditions, after a tedious but straightforward calculation, can be written in a matrix form which combined with the Bloch ansatz, furnish (see Refs. [9] and [17] for details)

$$
\cos (Q D)=(1 / 2) \operatorname{Tr}[T] \text {. }
$$

Here $Q$ is the Bloch wavevector of the collective excitation, and $D$ is the size of the superlattice unit cell. Eq. (5) follows from the fact that $T$ is an unimodular $2 \times 2$ matrix, and it describes the bulk modes of spin waves in a magnetic superlattice. Once we know the form of the transfer matrix $T$, the spin-wave spectra are determined.

\subsection{Semiconducting superlattice}

The second physical system that we consider in this article is the quasiperiodic semiconductor superlattices. To set up a quasiperiodic semiconductor structure, as before, we consider two different building blocks $A$ and $B$ which are arranged according to a quasiperiodic sequence. The layers $A$ (which model AlN) and $B$ (which model GaN) are characterized by the dielectric functions $\varepsilon_{A}(\omega)$ and $\varepsilon_{B}(\omega)$, and have thicknesses $d_{a}$ and $d_{b}$, respectively. We consider also optical-phonon propagation along the $x y$-plane, with the optical axis $c$ coinciding with the cartesian axis $z$. We use physical parameters modelling the nitride semiconductors AlN and GaN. Their hexagonal wurtzite structure allows the propagation of three bulk polar optical-phonons, among the nine optical-phonon modes, which are Raman and infrared active in the irreducible representation of $A_{1}(z)$ (z-axis) and $E_{1}(x y)$ ( $x y$-plane) at the $\Gamma$ point. Two of them are extraordinary waves associated with $z$ - and $x y$-polarized vibrations. The $z$-polarized mode has $A_{1}(z)$ symmetry, while the $x y$-polarized one has $E_{1}(x y)$ symmetry. The other one is an ordinary wave, which is always transverse and polarized in the $x y$-plane, with $E_{1}(x y)$ symmetry [18].

In our model, we consider, in each layer $A$ or $B$, a bulk wurtzite crystal, and our calculations are carried out within the framework of the dielectric continuum model. In this case, the fields associated with the optical modes in each layer should satisfy Maxwell's equations, i.e.,

$$
\begin{aligned}
& \vec{\nabla} \cdot \vec{D}_{j}=0, \\
& \vec{\nabla} \times \vec{H}_{j}=\frac{1}{c} \frac{\partial \vec{D}_{j}}{\partial t},
\end{aligned}
$$

where

$$
\vec{D}_{j}=\varepsilon_{j}(\omega) \vec{E}_{j}
$$


and $j$ specifies the nature of the layer (AlN or GaN). Due to the anisotropy of the medium, the dielectric tensor $\varepsilon(\omega)$ in each medium is given by

$$
\varepsilon(\omega)=\left(\begin{array}{ccc}
\varepsilon_{\perp}(\omega) & 0 & 0 \\
0 & \varepsilon_{\perp}(\omega) & 0 \\
0 & 0 & \varepsilon_{\|}(\omega)
\end{array}\right) .
$$

Here $\varepsilon_{\perp}(\omega)$ and $\varepsilon_{\|}(\omega)$ are the dielectric functions perpendicular and parallel to the $z$-axis, respectively. The explicit form of $\varepsilon_{\perp}(\omega)$ and $\varepsilon_{\|}(\omega)$ are given by

$$
\begin{aligned}
& \varepsilon_{\perp}(\omega)=\varepsilon_{\perp}(\infty) \frac{\omega^{2}-\omega_{L O, E_{1}}^{2}}{\omega^{2}-\omega_{T O, E_{1}}^{2}}, \\
& \varepsilon_{\|}(\omega)=\varepsilon_{\|}(\infty) \frac{\omega^{2}-\omega_{L O, A_{1}}^{2}}{\omega^{2}-\omega_{T O, A_{1}}^{2}} .
\end{aligned}
$$

Here $\varepsilon_{\perp}(\infty)\left(\varepsilon_{\|}(\infty)\right)$ is the high-frequency dielectric constant perpendicular (parallel) to the $z$-axis, and $\omega_{T O, X}$ $\left(\omega_{L O, X}\right)$, with $X=A_{1}(z)$ and $E_{1}(x y)$, is the transverse optical (longitudinal optical) phonon angular frequency for the mode $X$.

By solving the Maxwell equations, the electromagnetic fields associated to the optical phonon modes amplitudes, in a given layer $j=A$ or $B$, have an exponential plane-wave-like behavior. As in the spin-wave case, we follow the transfer-matrix approach, which is the mathematical prescription more appropriate to these kind of structures. The details of the calculations can be found elsewhere [19]. In both magnetic and semiconductor structures, the mathematical sequences applied to set up the systems, have a strong influence on the localization and scaling properties of the optical-phonon and spin-wave modes that can propagate. As a consequence, these sequences also have a strong influence on the contribution of these collective excitations to the specific heat behavior.

\subsection{Quasiperiodic sequences}

Let us briefly describe the quasiperiodic sequences considered here (for a review see Ref. [20]). The $N$ th generation of the Fibonacci sequence can be determined by appending the $(N-2)$ th generation to the $(N-1)$ th one, i.e., $S_{N}=S_{N-1} S_{N-2}(N>2)$. This algorithm construction requires initial conditions which are chosen to be $S_{0}=B$ and $S_{1}=A$. The Fibonacci generations can also be obtained by the substitution rules, $A \rightarrow A B, B \rightarrow A$. The Fibonacci generations are $S_{0}=[B], S_{1}=[A], S_{2}=[A B], S_{3}=[A B A], S_{4}=[A B A A B]$, etc. In a given generation $S_{N}$, the total number of letters is given by the Fibonacci number $F_{N}$, which is obtained by the relation $F_{N}=F_{N-1}+F_{N-2}$, with $F_{0}=F_{1}=1$. Also, $F_{N-1}$ and $F_{N-2}$ are the number of letters $A$ and $B$, respectively. As the generation order increases $(N \gg 1)$, the ratio $F_{N} / F_{N-1}$ approach to $\tau=(1+\sqrt{5}) / 2$, an irrational number which is known as the golden mean. On the other hand, the $N$ th generation of the double period sequence can be obtained from the relations, $S_{N}=S_{N-1} S_{N-1}^{\dagger}$, with $S_{N}^{\dagger}=$ $S_{N-1} S_{N-1}(N>2)$. The initial conditions are $S_{0}=A$ and $S_{1}=A B$. We can also use the substitution rules $A \rightarrow A B, B \rightarrow A A$. The double period generations are $S_{0}=[A], S_{1}=[A B], S_{2}=[A B A A]$, etc. Finally, the $N$ th generation of Thue-Morse sequence can be obtained from the relations, $S_{N}=S_{N-1} S_{N-1}^{\dagger}$, with $S_{N}^{\dagger}=$ $S_{N-1}^{\dagger} S_{N-1}(N>2)$. The initial conditions are $S_{0}=A$ and $S_{0}^{\dagger}=B$. We can also use the substitution rules $A \rightarrow A B, B \rightarrow B A$. The Thue-Morse generations are $S_{0}=[A], S_{1}=[A B], S_{2}=[A B B A]$, etc.

As we have explained in the previous sections, from an experimental perspective, a quasiperiodic superlattice is grown by juxtaposing the two materials $A$ and $B$ according to the above described sequences of letters. It can also be shown that the transfer matrices of consecutive generations of Fibonacci, double-period and Thue-Morse superlattices are related by convenient recursive relations (for details see Refs. $[9,17,19]$ ). It means that, from the knowledge of the transfer matrices $T_{S_{0}}$ and $T_{S_{1}}$, we can determine the transfer matrix for any generation and, consequently, the energy spectra of optical-phonon and spin-wave modes. 
a

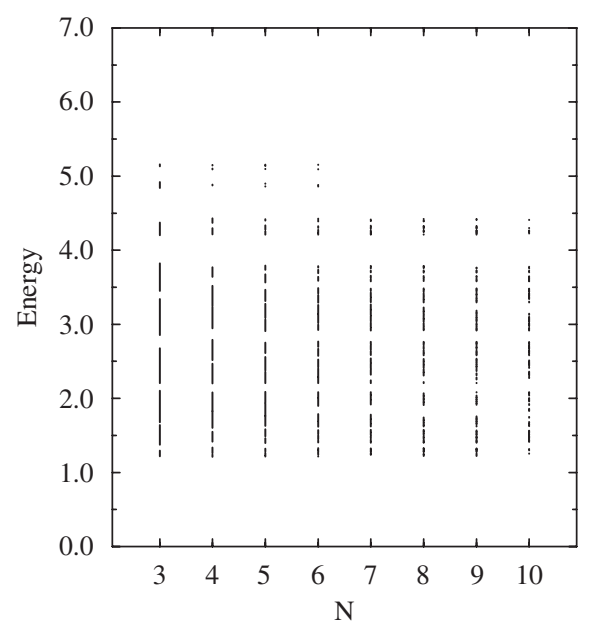

b

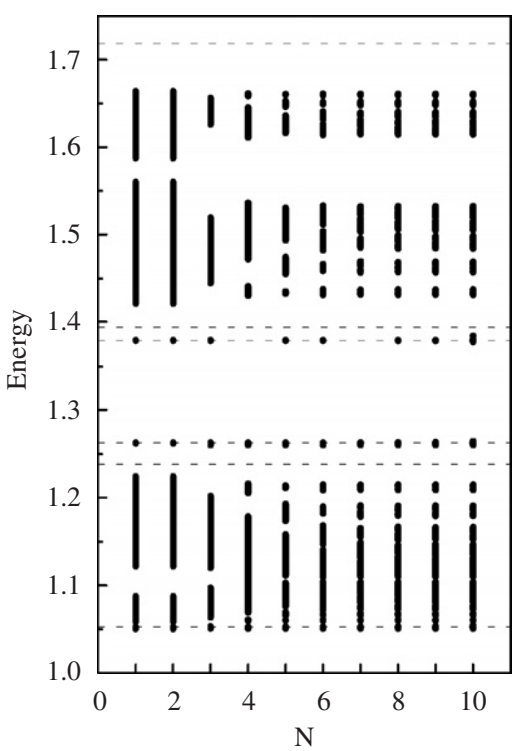

Fig. 1. The (a) spin-wave and (b) optical-phonon spectra for the Fibonacci case.

\section{Specific heat spectra}

We qualitatively illustrate in Fig. 1, for a fixed value of the in-plane wavevector, the spin-wave (Fig. 1(a)) and optical-phonon (Fig. 1(b)) energy spectra for Fibonacci superlattices versus the Fibonacci generation number $N$. One can see the forbidden and allowed energies which, as expected for large $N$, get narrower and narrower, presenting a typical Cantor set structure. A similar behavior can be observed for double period and Thue-Morse superlattices $[9,19]$.

In order to calculate the specific heat of any spectra similar to those one shown in Fig. 1, we have followed the lines of Ref. [15]. Let us briefly describe the procedure. In Fig. 1 each spectrum, for a fixed generation number $N$, has $M$ allowed continuous bands. We consider the level density within each band to be constant. The partition function for the $N$ th generation is then given by

$$
Z_{N}=\int_{0}^{\infty} \rho(\varepsilon) \mathrm{e}^{-\beta \varepsilon} \mathrm{d} \varepsilon
$$

Here $\beta=1 / T$ (by choosing the Boltzmann's constant $k_{B}=1$ ), and we take the density of states $\rho(\varepsilon)=1$. After a straightforward calculation we can write $Z_{N}$ as

$$
Z_{N}=\frac{1}{\beta} \sum_{i=1,3, \ldots}^{2 M-1} \mathrm{e}^{-\beta \varepsilon_{i}}\left(1-\mathrm{e}^{-\beta \Delta_{i}}\right) .
$$

Here the subscript $N$ is the generation number, $M$ is the number of allowed bands and $\Delta_{i}=\varepsilon_{i+1}-\varepsilon_{i}$ is the difference between the top and bottom energy levels of each band. The specific heat is then given by

$$
C_{N}(T)=\frac{\partial}{\partial T}\left(T^{2} \frac{\partial \ln Z_{N}}{\partial T}\right)
$$

which can be written as

$$
C_{N}(T)=1+\frac{\beta f_{N}}{Z_{N}}-\frac{g_{N}^{2}}{Z_{N}^{2}} .
$$


Here

$$
f_{N}=\sum_{i=1,3, \ldots}^{2 M-1}\left(\varepsilon_{i}^{2} \mathrm{e}^{-\beta \varepsilon_{i}}-\varepsilon_{i+1}^{2} \mathrm{e}^{-\beta \varepsilon_{i+1}}\right)
$$

and

$$
g_{N}=\sum_{i=1,3, \ldots}^{2 M-1}\left(\varepsilon_{i} \mathrm{e}^{-\beta \varepsilon_{i}}-\varepsilon_{i+1} \mathrm{e}^{-\beta \varepsilon_{i+1}}\right) .
$$

Therefore, once we know the energy spectra of the excitations which propagates in a given sequence's generation of a quasiperiodic structure, we can numerically determine the associated specific heat by using (15).

\section{Numerical results and discussions}

Let us now discuss our numerical results. We should remark that in this section we do not show the results for the Fibonacci magnetic superlattices because they can be found elsewhere $[15,16]$. In Fig. 2, we show the $\log -\log$ plot of the specific heat spectra of the Fibonacci semiconductor superlattices. Their profiles define clearly two classes of oscillations: one for the even and the other for the odd generation numbers of the sequence. We also conclude that the number of oscillations observed in the specific heat spectra is related to the generation number $N$ (more oscillations appear as $N$ increases). One can see that the amplitude of the odd oscillations is bigger than the amplitude of the even ones (except for $N=3$ because the quasiperiodicity is not strong enough). It is interesting to remark that a quite similar behavior is found for the specific heat of Fibonacci magnetic superlattices [15,16]. It means that, although we are dealing with different physical systems, the stacking pattern strongly influence the physical properties, which is reflected in the specific heat behavior.

In Fig. 3, we show the specific heat for the double-period superlattices. In Fig. 3(a) it is shown the specific heat behavior for the double-period magnetic superlattices. As in the Fibonacci superlattices, there are oscillations although they do not have the same standard of behavior met in the Fibonacci case, i.e., two groups of oscillations corresponding to even and odd generations. A similar behavior is also found for the specific heat of double-period semiconductor superlattices. This is shown in Fig. 3(b). It is interesting to note

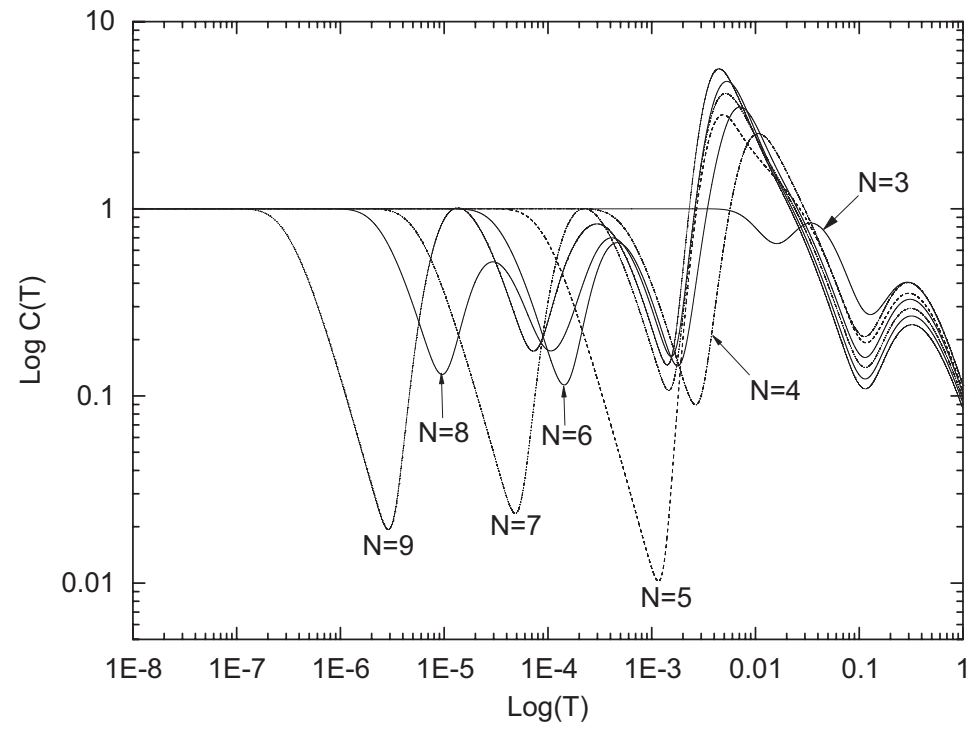

Fig. 2. Log-log plot of the specific heat versus temperature for the Fibonacci generations of the quasiperiodic semiconductor superlattice. Observe a different behavior for the even $(N=4,6$, and 8$)$ and odd $(N=5,7$, and 9$)$ generation numbers. 
a

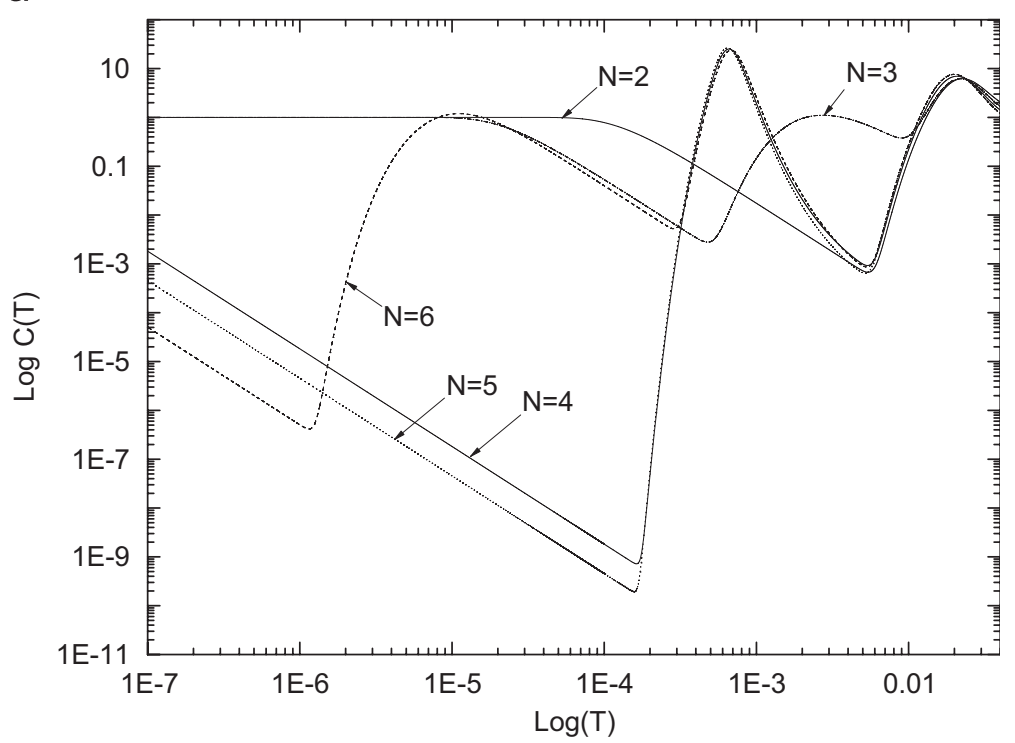

b

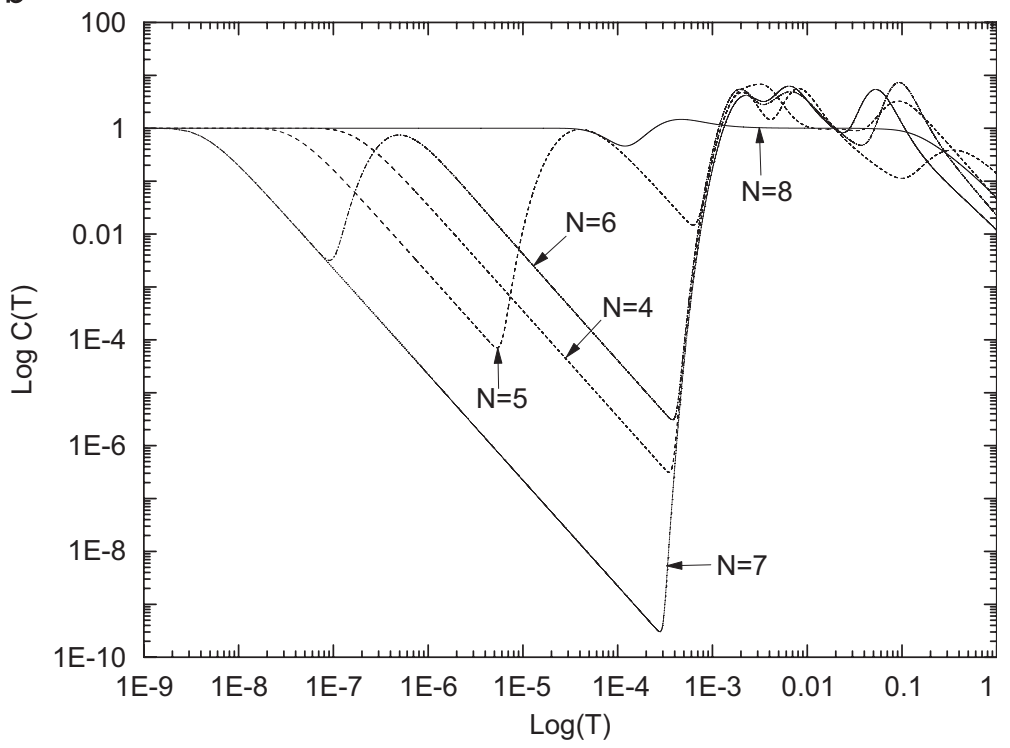

Fig. 3. Log-log plot of the specific heat versus temperature for the double-period generations of the quasiperiodic (a) magnetic and (b) semiconductor superlattices. For this case there is no connection between the generation number $N$ and the number of oscillations.

that, different from the Fibonacci case, there is no relation between the number of oscillations and the generation number $N$. Besides, the pattern of the oscillations is more disordered than the Fibonacci one. This can be considered as a consequence of the fact that the double-period sequence is more disordered than the Fibonacci sequence, once they present different Fourier spectrum (dense pure point for Fibonacci and singular continuous for double-period). However, as in the Fibonacci case, we can see that both double-period systems present the same standard of behavior for the specific heat. We can infer that the stacking pattern is again determinant for the oscillation pattern of the specific heat.

Our analysis would not be complete without the discussion of the specific heat behavior of the Thue-Morse superlattices. This case is shown in Fig. 4. We should remark that it was very difficult numerically to evaluate the energy spectra for Thue-Morse superlattices, because the localization of the energy bands is very strong 


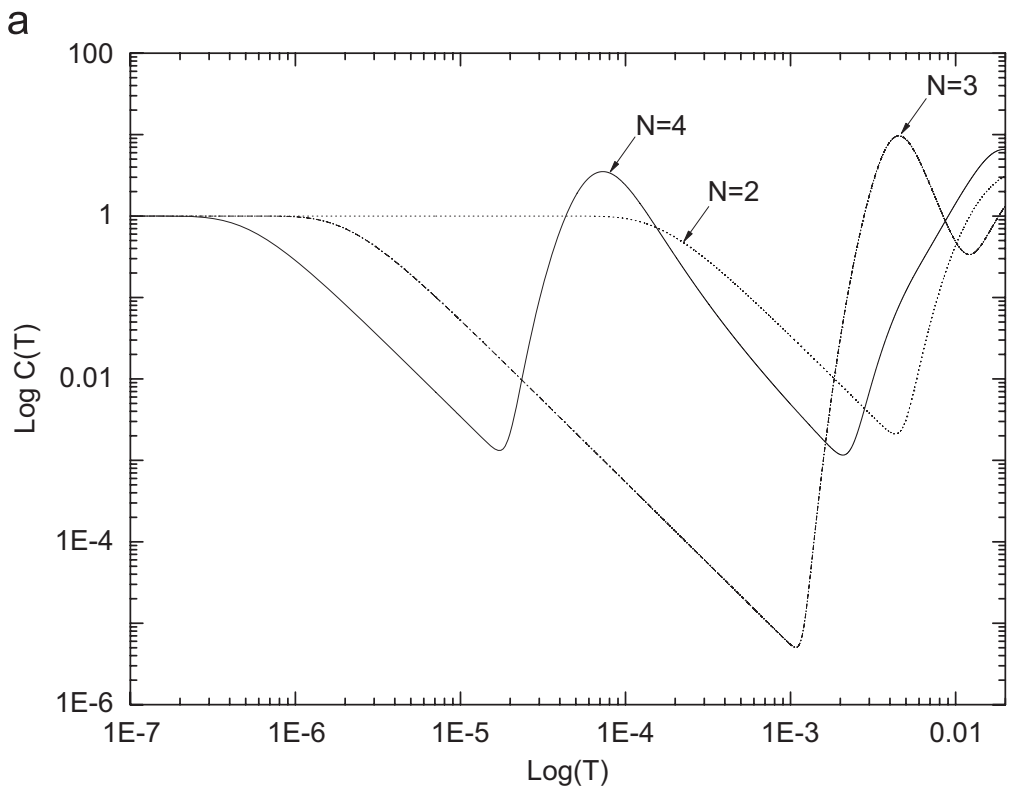

Fig. 4. Log-log plot of the specific heat versus temperature for Thue-Morse generations of the quasiperiodic (a) magnetic and (b) semiconductor superlattices. As for the double-period case, there is no connection between the generation number $N$ and the number of oscillations.

for this case. This strong localization for Thue-Morse structures is known from the literature [20] and it is attributed to the higher disorder degree of Thue-Morse sequence. However, we believe that we can infer the qualitative behavior of the specific heat of Thue-Morse structures with the present numerical results. Thus, in Fig. 4(a) we present the specific heat of three generations of Thue-Morse magnetic superlattices. As in the two previous cases, the specific heat present oscillations, although their pattern is different from Fibonacci and double-period superlattices. However, for Thue-Morse case, the oscillations are more similar to the double-period case than to the Fibonacci one. Fig. 4(b) shows the specific heat for Thue-Morse semiconductor superlattices. Once again the standard of oscillations resembles very much that of Fig. 4(a). 
As in the double-period case, there is no relation between the number of oscillations and the generation number $N$. One can also see that the pattern of the oscillations of the specific heat of Thue-Morse superlattices is even more disordered than the double-period one. This can be explained because, although double-period and Thue-Morse sequences present the same Fourier spectrum (singular continuous), Thue-Morse sequence is considered more disordered than double-period sequence. Therefore, this is reflected in the specific heat behavior.

The origin of the oscillations shown in Figs. 2-4 is associated to the fractal spectra of energy of the quasiperiodic structures. As we can see from Fig. 1, the spectra of energy is highly fragmented where the allowed energies are separated by gaps. The distribution of both allowed energies and gaps depends on the stacking pattern, i.e., on the quasiperiodic sequence applied in the construction of the structure. The consequence of the existence of gaps is the presence of oscillations in the specific heat. In fact, the oscillation pattern is composed by the superposition of Schottky anomalies each one corresponding to each one of the scales of the fractal spectra of energy [13].

Before concluding, let us comment on the wavelike nature of both elementary excitations (spin waves and phonons). It is shown in Figs. 2-4 that the oscillation pattern is qualitatively the same, no matter the excitation, for a given quasiperiodic structure. This is a very reasonable result because each quasiperiodic structure presents its own stacking pattern. A given stacking pattern means a well defined sequence of interfaces in the unit cell and, therefore, in the whole superlattice. At the interfaces part of the propagating wave is reflected and part is transmitted, what defines the features of the energy spectra. Thus, quasiperiodic structures, constructed according to the same quasiperiodic sequence, exhibit virtually the same oscillation pattern of the specific heat because their energy spectra present similar fractal properties.

To summarize, we have investigated in this paper the specific heat contribution, from the energy spectra of optical-phonon and spin-wave modes, in quasicrystals of the Fibonacci, double-period and Thue-Morse types. We have shown, as a common aspect of the model employed here, that the specific heat presents oscillations for all quasiperiodic sequences considered. These oscillations are consequences that the specific heat is a log-periodic function of the temperature and are associated to the fractal spectra of energy of the quasiperiodic structures. They can also be defined as the signatures or fingerprints of each quasiperiodic system no matter the excitation, once both phenomena present a wavelike nature. We show that the doubleperiod and Thue-Morse specific heat spectra present a non-ordered pattern of oscillations, which is quite different from the Fibonacci one. Therefore, apart from the common presence of oscillations in the specific heat, there is no other connection between Fibonacci case and Thue-Morse and double-period cases considered in this work. Finally, we can also conclude that the pattern of oscillations strongly depends on the degree of disorder of the sequence used in the stacking pattern.

\section{Acknowledgment}

We would like to thank the Brazilian Research Council CNPq.

\section{References}

[1] D. Shechtman, I. Blech, D. Gratias, J.W. Cahn, Phys. Rev. Lett. 53 (1984) 1951.

[2] P.J. Steinhardt, S. Ostlund, The Physics of Quasicrystals, World Scientific, Singapore, 1987.

[3] C. Janot, Quasicrystals: A Primer, Oxford University Press, Oxford, 1993.

[4] M. Senechal, Quasicrystals and Geometry, Cambridge University Press, Cambridge, 1995.

[5] M.S. Vasconcelos, E.L. Albuquerque, A.M. Mariz, J. Phys.: Condens. Matter 10 (1998) 5839.

[6] M. Quilichini, T. Janssen, Rev. Mod. Phys. 69 (1997) 277.

[7] P.M.C. de Oliveira, E.L. Albuquerque, A.M. Mariz, Physica A 227 (1996) 206.

[8] M.S. Vasconcelos, E.L. Albuquerque, Phys. Rev. B 57 (1998) 2826.

[9] C.G. Bezerra, E.L. Albuquerque, Physica A 245 (1997) 379; C.G. Bezerra, E.L. Albuquerque, Physica A 255 (1998) 285.

[10] D.H.A.L. Anselmo, M.G. Cottam, E.L. Albuquerque, J. Phys.: Condens. Matter 12 (2000) 1041.

[11] M.S. Vasconcelos, E.L. Albuquerque, Solid State Commun. 117 (2001) 495.

[12] R. Merlin, K. Bajema, R. Clarke, K.M. Mohanty, J.D. Axe, Phys. Rev. Lett. 57 (1986) 1157. 
[13] C. Tsallis, L.R. da Silva, R.S. Mendes, R.O. Vallejos, A.M. Mariz, Phys. Rev. E 56 (1997) R4922.

[14] A.V. Coronado, P. Carpena, Phys. Rev. E 73 (2006) 016124.

[15] C.G. Bezerra, E.L. Albuquerque, A.M. Mariz, L.R. da Silva, C. Tsallis, Physica A 294 (2001) 415.

[16] C.G. Bezerra, E.L. Albuquerque, M.G. Cottam, Physica A 301 (2001) 341.

[17] C.G. Bezerra, M.G. Cottam, Phys. Rev. B 65 (2002) 054412.

[18] M.A. Stroscio, M. Dutta, Phonons in Nanostructures, Cambridge University Press, Cambridge, 2001.

[19] D.H.A.L. Anselmo, A.L. Dantas, S.K. Medeiros, E.L. Albuquerque, V.N. Freire, Physica A 349 (2005) 259.

[20] E.L. Albuquerque, M.G. Cottam, Phys. Rep. 376 (2003) 225. 